УДK 517.962 .2

Г. П. ПелюО (Ин-г математики НАН Укранны, Киеß)

\title{
О СТРУКТУРЕ ОБЩЕГО РЕШЕНИЯ СИСТЕМ НЕЛИНЕЙНЫХ РАЗНОСТНЫХ УРАВНЕНИЙ
}

We investigate the structure of general solution of systems of nonlinear difference equations with continuous argument in a neighborhood of equilibrium.

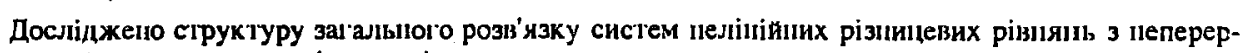
винм аргумегтом в околі стану різиоваги.

Рассмотрим систему нелинейных разностных уравнений

$$
x(t+1)=\Lambda x(t)+f(t, x(t)),
$$

где $t \in R=(-\infty,+\infty), \Lambda$ - вешественная, постоянная $(n \times n)$-мерная матрица, $f: R \times C^{n} \rightarrow C^{n}, x(t)$ - неизвестная комплекснозначная вектор-функция размерности $n$. Будем исследовать структуру множества непрерывных решений этой системы, находящихся в окрестности ее тривиального решения $(f(t, 0) \equiv 0)$. При различных предположениях эта задача изучалась многими математиками. В частности, для широких классов таких уравнений построено представление общего непрерывного решения [1 - 6]. Продолжая начатые в [5, 6] исследования, в настоящей работе удалось получить аналогичные результаты при менее обременительных предположениях относительно вектор-функции $f(t, x)$.

1. Общее решенне снстемы нелинейных разностных уравненнй (1) в окрестности ее тривиального решения $x(t) \equiv 0$. Рассмотрим систему уравнении (1) при следующих предположениях:

1) собственные числа $\lambda_{i}, i=1, \ldots, n$, матрицы $\Lambda$ вещественны и удовлетворяют соотношениям

$$
\lambda_{i} \neq \lambda_{j}, \quad i \neq j, \quad 0<\left|\lambda_{i}\right|<1, \quad i, j=1, \ldots, n
$$

2) $\lambda_{*}^{-1} \lambda^{*+\alpha}<1$, где $\lambda_{*}=\min \left\{\left|\lambda_{i}\right|, i=1, \ldots, n\right\}, \lambda^{*}=\max \left\{\left|\lambda_{i}\right|, i=1, \ldots, n\right\}$;

3) вектор-функция $f(t, x)=\left(f_{1}(t, x), \ldots, f_{n}(t, x)\right)$ является непрерывной и ограниченно по $t$, непрерывно дифференцируемой по $x$ при $t \in R$, $|x|=\max _{1 \leq i \leq n}\left|x_{i}\right| \leq b$ и

$$
f(t, 0) \equiv 0,\left.\quad \frac{\partial f(t, x)}{\partial x}\right|_{x=0} \equiv 0, \quad \text { где } \frac{\partial f(t, x)}{\partial x}=\left(\frac{\partial f_{i}(t, x)}{\partial x_{j}}\right)
$$

4) $\left|\frac{\partial f(t, x)}{\partial x}-\frac{\partial f(t, y)}{\partial y}\right| \leq L|x-y|^{\alpha}$, где

$$
\begin{gathered}
\left|\frac{\partial f(t, x)}{\partial x}\right|=\max _{1 \leq i \leq n} \sum_{j=1}^{n}\left|\frac{\partial f_{i}(t, x)}{\partial x_{j}}\right|, \\
L=\text { const }>0,0<\alpha \leq 1,(t, x),(t, y) \in D=R \times[-b, b] .
\end{gathered}
$$

Поскольку в силу условия 1 существует неособая замена переменных $x(t)=$ $=C y(t)$, приводящая систему уравненин (1) к виду

$$
y(t+1)=C^{-1} \Lambda C y(t)+C^{-1} f(t, C y(t)),
$$


причем $C^{-1} \Lambda C=\operatorname{diag}\left(\lambda_{1}, \ldots, \lambda_{n}\right)$, то в дальнейшем будем считать, что сама матрица $\Lambda$ имеет такой вид, т. е. $\Lambda=\operatorname{diag}\left(\lambda_{1}, \ldots, \lambda_{n}\right)$.

Теорема 1. Пусть выполняются условия 1 - 4. Тогда существует замена перехіенных

$$
y(t)=x(t)+\gamma(t, x(t)),
$$

где вектор-функция $\gamma(t, x)=\left(\gamma_{1}(t, x), \ldots, \gamma_{n}(t, x)\right)$ является непрерывной $и$ ограниченной по $t$, непрерывно дифференцируельой по $x$ в некоторой области $D_{*}=\boldsymbol{R} \times\left[-b_{*}, b_{*}\right], b_{*}<b$, и такой, что выполняотся соотночения

$$
\begin{gathered}
\gamma(t, 0) \equiv 0,\left.\frac{\partial \gamma(t, x)}{\partial x}\right|_{x=0} \equiv 0, \\
\left|\frac{\partial \gamma(t, x)}{\partial x}-\frac{\partial \gamma(t, y)}{\partial y}\right| \leq M|x-y|^{\alpha}, \quad M=\text { const }>0, \quad(t, x),(t, y) \in D_{*} ;
\end{gathered}
$$

приводящая систеяу уравнений (1) к линейному виду

$$
y(t+1)=\Lambda y(t) .
$$

Для доказательства теоремы достаточно, очевидно, показать, что существует решение системы уравнений

$$
\gamma(t+1, \Lambda x+f(t, x))=\Lambda \gamma(t, x)-f(t, x),
$$

удовлетворяющее указанным в теореме условиям.

С помощью соотношений

$$
\begin{gathered}
\gamma_{0}(t, x)=0, \\
\gamma_{m}(t, x)=\Lambda^{-1} \gamma_{m-1}(t+1, \Lambda x+f(t, x))+\Lambda^{-1} f(t, x), \quad m=1,2, \ldots,
\end{gathered}
$$

определим последовательность вектор-функции $\left\{\gamma_{m}(t, x)\right\}$ и докажем, что в некоторой области $D * \subset D$ она равномерно сходится к вектор-функции $\gamma(t, x)$, которая удовлетворяет указанным в теореме условиям и является решением системы уравнений (5).

Сначала покажем, что при достаточно малом $b_{*}<b$ и всех $m=1,2, \ldots$ выполняются оценки

$$
\begin{gathered}
\left|\gamma_{m}(t, x)-\gamma_{m-1}(t, x)\right| \leq M_{0} \theta^{m-1}|x|^{1+\alpha}, \\
\left|\frac{\partial \gamma_{m}(t, x)}{\partial x}-\frac{\partial \gamma_{m-1}(t, x)}{\partial x}\right| \leq M_{0} \theta^{m-1}|x|^{\alpha}, \\
\left|\frac{\partial \gamma_{m}(t, x)}{\partial x}-\frac{\partial \gamma_{m}(t, y)}{\partial y}\right| \leq M_{1}|x-y|^{\alpha}
\end{gathered}
$$

где $M_{0}, M_{1}$ - положительные постоянные $\left(M_{0}, M_{1}>\lambda_{*}^{-1} L\right), \lambda_{*}^{-1} \lambda^{*^{1+\alpha}}<\theta<1$, $(t, x),(t, y) \in D_{*}$.

В самом деле, поскольку

$$
\gamma_{1}(t, x)-\gamma_{0}(t, x)=\Lambda^{-1} f(t, x),
$$

то в силу условий 3,4 оценки (7) выполняются при $m=1$. Рассуждая по индукции, предположим справедливость оценок (7) для некоторого $m \geq 1$ и докажем, что они сохраняются при переходе от $m \times m+1$. Действительно, поскольку при $t \in \boldsymbol{R},|x| \leq b$, имеем (вытекает из условий 3,4 ) 


$$
|\Lambda x+f(t, x)| \leq\left(\lambda^{*}+\delta\right)|x|, \quad\left|\Lambda+\frac{\partial f(t, x)}{\partial x}\right| \leq \lambda^{*}+\delta,
$$

где $\delta=\delta\left(b_{*}\right) \rightarrow 0$ при $b_{*} \rightarrow 0$, то, принимая во внимание соотношения

$$
\begin{gathered}
\gamma_{m+1}(t, x)-\gamma_{m}(t, x)=\Lambda^{-1}\left[\gamma_{m}(t+1, \Lambda x+f(t, x))-\right. \\
\left.-\gamma_{m-1}(t+1, \Lambda x+f(t, x))\right] \\
\frac{\partial \gamma_{m+1}(t, x)}{\partial x}-\frac{\partial \gamma_{m}(t, x)}{\partial x}=\Lambda^{-1}\left[\frac{\partial \gamma_{m}}{\partial x}(t+1, \Lambda x+f(t, x))-\right. \\
\left.-\frac{\partial \gamma_{m-1}(t, x)}{\partial x}(t+1, \Lambda x+f(t, x))\right]\left(\Lambda+\frac{\partial f(t, x)}{\partial x}\right),
\end{gathered}
$$

находим

$$
\begin{aligned}
& \left|\gamma_{m+1}(t, x)-\gamma_{m}(t, x)\right| \leq \lambda_{*}^{-1} \mid \gamma_{m}(t+1, \Lambda x+f(t, x))- \\
& -\gamma_{m-1}(t+1, \Lambda x+f(t, x))\left|\leq \lambda_{*}^{-1} M_{0} \theta^{m-1}\right| \Lambda x+\left.f(t, x)\right|^{1+\alpha} \leq \\
& \leq M_{0} \theta^{m-1} \lambda_{*}^{-1}\left(\lambda^{*}+\delta\right)^{1+\alpha}|x|^{1+\alpha}, \\
& \left|\frac{\partial \gamma_{m+1}(t, x)}{\partial x}-\frac{\partial \gamma_{m}(t, x)}{\partial x}\right| \leq \lambda_{*}^{-1} \mid \frac{\partial \gamma_{m}}{\partial x}(t+1, \Lambda x+f(t, x))- \\
& -\frac{\partial \gamma_{m-1}}{\partial x}(t+1, \Lambda x+f(t, x))|| \Lambda+\frac{\partial f(t, x)}{\partial x} \mid \leq \\
& \leq \lambda_{*}^{-1} M_{0} \theta^{m-1}|\Lambda x+f(t, x)|^{\alpha}\left(\lambda^{*}+\delta\right) \leq M_{0} \theta^{m-1} \lambda_{*}^{-1}\left(\lambda^{*}+\delta\right)^{1+\alpha}|x|^{\alpha}, \\
& \left|\frac{\partial \gamma_{m+1}(t, x)}{\partial x}-\frac{\partial \gamma_{m+1}(t, y)}{\partial y}\right| \leq \lambda_{*}^{-1} \mid \frac{\partial \gamma_{m}}{\partial x}(t+1, \Lambda x+f(t, x))\left(\Lambda+\frac{\partial f(t, x)}{\partial x}\right)- \\
& -\frac{\partial \gamma_{m}}{\partial y}(t+1, \Lambda y+f(t, y))\left(\Lambda+\frac{\partial f(t, y)}{\partial y}\right)\left|+\lambda_{*}^{-1}\right| \frac{\partial f(t, x)}{\partial x}-\frac{\partial f(t, y)}{\partial y} \mid \leq \\
& \leq \lambda_{*}^{-1}\left|\frac{\partial \gamma_{m}}{\partial x}(t+1, \Lambda x+f(t, x))-\frac{\partial \gamma_{m}}{\partial y}(t+1, \Lambda y+f(t, y))\right|\left|\Lambda+\frac{\partial f(t, x)}{\partial x}\right|+ \\
& +\lambda_{*}^{-1}\left|\frac{\partial \gamma_{m}}{\partial y}(t+1, \Lambda y+f(t, y))\right|\left|\frac{\partial f(t, x)}{\partial x}-\frac{\partial f(t, y)}{\partial y}\right|+\lambda_{*}^{-1}\left|\frac{\partial f(t, x)}{\partial x}-\frac{\partial f(t, y)}{\partial y}\right| \leq \\
& \leq \lambda_{*}^{-1} M_{1}|\Lambda x+f(t, x)-\Lambda y-f(t, y)|^{\alpha}\left|\Lambda+\frac{\partial f(t, x)}{\partial x}\right|+\lambda_{*}^{-1} \frac{M_{0}}{1-\theta}\left(\lambda^{*}+\delta\right)|y|^{\alpha} \times \\
& \times L|x-y|^{\alpha}+\lambda_{*}^{-1} L|x-y|^{\alpha} \leq M_{1} \lambda_{*}^{-1}\left(\lambda^{*}+\delta\right)^{1+\alpha}|x-y|^{\alpha}+\frac{M_{0}}{1-\theta} L \lambda_{*}^{-1}\left(\lambda^{*}+\right. \\
& +\delta) b_{*}^{\alpha}|x-y|^{\alpha}+\lambda_{*}^{-1} L|x-y|^{\alpha}=M_{1}\left[\lambda_{*}^{-1}\left(\lambda^{*}+\delta\right)^{1+\alpha}+\right. \\
& \left.+\frac{\lambda_{*}^{-1}\left(\lambda^{*}+\delta\right)}{1-\theta} M_{0} L M_{1}^{-1} b_{*}^{\alpha}+\lambda_{*}^{-1} L M_{1}^{-1}\right]|x-y|^{\alpha} \text {. }
\end{aligned}
$$

Tax ках $\lambda_{*}^{-1} \lambda^{*+\alpha}<\theta$, то при достаточно малом $b_{*}$ и достаточно большом $M_{1}$ имeem 


$$
\begin{gathered}
\lambda_{*}^{-1}\left(\lambda^{*}+\delta\right)^{1+\alpha}<\theta, \\
\lambda_{*}^{-1}\left(\lambda^{*}+\delta\right)^{1+\alpha}+\frac{\lambda_{*}^{-1}\left(\lambda^{*}+\delta\right)}{1-\theta} M_{0} L M_{1}^{-1} b_{*}^{\alpha}+\lambda_{*}^{-1} L M_{1}^{-1} \leq 1,
\end{gathered}
$$

и, следовательно, выполняются соотношения

$$
\begin{gathered}
\left|\gamma_{m+1}(t, x)-\gamma_{m}(t, x)\right| \leq M_{0} \theta^{m}|x|^{1+\alpha}, \\
\left|\frac{\partial \gamma_{m+1}(t, x)}{\partial x}-\frac{\partial \gamma_{m}(t, x)}{\partial x}\right| \leq M_{0} \theta^{m}|x|^{\alpha}, \\
\left|\frac{\partial \gamma_{m+1}(t, x)}{\partial x}-\frac{\partial \gamma_{m+1}(t, y)}{\partial y}\right| \leq M_{1}|x-y|^{\alpha} .
\end{gathered}
$$

Тем самым доказано, что оценки (7) выполняются при $(t, x),(t, y) \in D *$ и всех $m \geq 1$.

Из (7) непосредственно вытекает, что последовательность вектор-функций $\gamma_{m}(t, x), m=0,1, \ldots$, определенных соотношениями (6), равномерно сходится при $(t, x) \in D *$ к вектор-функции $\gamma(t, x)$, которая в области $D_{*}$ является непрерывной и ограниченной по $t$, непрерывно дифференцируемой по $x$ и удовлетворяет условиям (3). Переходя в (6) к пределу при $m \rightarrow \infty$, можно убедиться, что вектор-функция $\gamma(t, x)=\lim _{m \rightarrow \infty} \gamma_{m}(t, x)$ является решеннем системы уравнениम (5). Теорема 1 доказана.

Замечание 1. Теорема 1 справедлива и в случае. когда среди собственных чисел $\lambda_{i}, i=1, \ldots, n$, имеются комплексные.

Используя теорему 1 , можно получить представление любого непрерывного решения системы уравнений (1) в окрестности тривиального решения. Действительно, поскольку общее непрерывное решение системы уравненин (4) имеет вид

$$
y_{i}(t)=\left|\lambda_{i}\right|^{t} \omega_{i}(t), \quad i=1, \ldots, n,
$$

где $\omega_{i}(t)$ - проиэвольные непрерывные функции, удовлетворяющие условиям $\omega_{i}(t+1)=\operatorname{sign} \lambda_{i} \omega_{i}(t), i=1, \ldots, n$, то (вытекает из (2) и (3)) для произвольного непрерывного решения системы уравнении (1), удовлетворяющего при $t \geq 0$ условию $|x(t)| \leq b_{*}$, получаем

$$
x(t)=y(t)+\gamma^{-1}(t, y(t)),
$$

где $y(t)=\left(\left|\lambda_{1}\right|^{t} \omega_{1}(t), \ldots,\left|\lambda_{n}\right|^{t} \omega_{n}(t)\right)$ и $\gamma^{-1}(t, y)$ - некоторая непрерывная и ограниченная по $t$, непрерывно дифференцируемая по у в некоторон области $R \times[-\tilde{b}, \tilde{b}], \tilde{b}<b_{*}$, вектор-функция, удовлетворяющая условиям (3), и $\omega_{i}(t)$, $i=1, \ldots, n-$ некоторые непрерывные функции, удовлетворяюшие условиям $\omega_{i}(t+1)=\operatorname{sign} \lambda_{i} \omega_{i}(t), i=1, \ldots, n$.

2. Инвариантные многообразия систем нелинейых разностных уравнений и их своһ̆ства. Рассмотрим теперь случай, когда нарушаются условия 1 , 2. Именно, предположим, что собственные числа $\lambda_{i}, i=1, \ldots, n$, матрицы $\Lambda$ удовлетворяют условиям:

$$
\begin{aligned}
& \text { 1') } \lambda_{i} \neq \lambda_{j}, \quad i \neq j, \quad i, j=1, \ldots, n, \quad 0<\left|\lambda_{i}\right|<1<\left|\lambda_{j}\right| \text {, } \\
& i=1, \ldots, p, j=p+1, \ldots, n \text {; } \\
& \left.2^{\prime}\right) \quad \lambda_{*}^{-1} \lambda^{*^{1+\alpha}}<1 \text {, где } \tilde{\lambda}_{*}=\min \left\{\left|\lambda_{i}\right| i=1, \ldots, p\right\}, \quad \tilde{\lambda}^{*}=\max \left\{\left|\lambda_{i}\right|,\right.
\end{aligned}
$$


Для удобства систему уравнений (1) запишем в виде

$$
\begin{aligned}
& x(t+1)=\tilde{\Lambda} x(t)+\tilde{f}(t, x(t), y(t)), \\
& y(t+1)=\tilde{\tilde{\Lambda}} x(t)+\tilde{\tilde{f}}(t, x(t), y(t)),
\end{aligned}
$$

где $\bar{\Lambda}=\operatorname{diag}\left\{\lambda_{1}, \ldots, \lambda_{p}\right\}, \tilde{\Lambda}=\operatorname{diag}\left\{\lambda_{p+1}, \ldots, \lambda_{n}\right\}, \quad x=\left(x_{1}, \ldots, x_{p}\right), \quad y=\left(y_{p+1}, \ldots\right.$ $\left.\ldots, y_{n}\right), \tilde{f}=\left(f_{1}, \ldots, f_{p}\right), \quad \overline{\bar{f}}=\left(f_{p+1}, \ldots, f_{n}\right)$, и предположим, что вектор-функции $\tilde{f}(t, x, y), \quad \tilde{\tilde{f}}(t, x, y)$ удовлетворяют условиям 3,4 . Как и прежде, нашей конечной целью является построение представления общего непрерывного решения системы (10) в окрестности тривиального решения $x(t)=0, y(t)=0$. Поскольку в этом случае теорема 1, вообще говоря, не имеет места, то получить представление общего непрерывного решения вида (9) не представляется возможным. Однако в этом случае справедлива следующая теорема, которая дает возможность существенно упростить исследование системы уравнений (10).

Теорема 2. Пусть өыполняются условия 1', 2', 3, 4. Тогда существует зальена перельеных

$$
x(t)=\bar{x}(t), \quad y(t)=\bar{y}(t)+\psi(t, \bar{x}(t))
$$

такая, что (n-p)-льерная вектор-функция $\psi(t, \bar{x}(t))$ является непрерывной и ограниченной по $t$, непрерывно дифференцируелюй по $\bar{x}$ в некоторой области $D_{*}=\boldsymbol{R} \times\left[-b_{*}, b_{*}\right], b_{*}<b$, удовлетворяет условияль

$$
\begin{gathered}
\psi(t, 0) \equiv 0,\left.\quad \frac{\partial \psi(t, \bar{x})}{\partial \bar{x}}\right|_{\bar{x}=0} \equiv 0, \\
\left|\frac{\partial \psi(t, \bar{x})}{\partial \bar{x}}-\frac{\partial \psi(t, \bar{y})}{\partial \bar{y}}\right| \leq M|\bar{x}-\bar{y}|^{\alpha}, \quad M=\text { const }>0,
\end{gathered}
$$

$(t, \bar{x}),(t, \bar{y}) \in D_{*}$, и в новых переменных $\bar{x}, \bar{y}$ систельа уравнений (10) ильеет вид

$$
\begin{aligned}
& \bar{x}(t+1)=\tilde{\Lambda} \bar{x}(t)+\bar{f}(t, \bar{x}(t), \bar{y}(t)), \\
& \bar{y}(t+1)=\tilde{\tilde{\Lambda}} \bar{y}(t)+\overline{\bar{f}}(t, \bar{x}(t), \bar{y}(t)),
\end{aligned}
$$

где вектор-функции $\bar{f}(t, \bar{x}, \bar{y}), \quad \overline{\tilde{f}}(t, \bar{x}, \bar{y})$ удовлетворяют условияль 3.4 и $\overline{\bar{f}}(t, \bar{x}, 0) \equiv 0$.

Замечание 2. Условие $\overline{\bar{f}}(t, \bar{x}, 0) \equiv 0$ означает, что многообразие $y=\psi(t, x)$ является локально инвариантным относительно отображения

$$
\begin{gathered}
x \rightarrow \tilde{\Lambda} x+\tilde{f}(t, x, y), \\
y \rightarrow \tilde{\tilde{\Lambda}} y+\tilde{\tilde{f}}(t, x, y), \\
t \rightarrow t+1 .
\end{gathered}
$$

Доказательство теоремы 2. Выполняя в (10) замену переменных (11), получаем (13), где

$$
\bar{f}(t, \bar{x}(t), \bar{y}(t))=\bar{f}(t, \bar{x}(t), \bar{y}(t)+\psi(t, \bar{x}(t))),
$$




$$
\begin{gathered}
\overline{\bar{f}}(t, \bar{x}(t), \bar{y}(t))=\tilde{\tilde{f}}(t, \bar{x}(t), \bar{y}(t)+\psi(t, \bar{x}(t)))+\tilde{\tilde{\Lambda}} \psi(t, \bar{x}(t))- \\
-\psi(t+1, \tilde{\Lambda} \bar{x}(t)+\tilde{f}(t, \bar{x}(t), \bar{y}(t)+\psi(t, \bar{x}(t)))) .
\end{gathered}
$$

Отсюда непосредственно вытекает, что для доказательства теоремы достаточно доказать существование решения системы функциональных уравнений

$$
\psi(t+1, \bar{\Lambda} \bar{x}+\tilde{f}(t, \bar{x}, \psi(t, \bar{x})))=\tilde{\tilde{\Lambda}} \psi(t, \bar{x})+\tilde{\tilde{f}}(t, \bar{x}, \psi(t, \bar{x}))
$$

с указанными в теореме свойствами.

С помощью соотношений

$$
\begin{gathered}
\Psi_{0}(t, \bar{x})=0, \\
\psi_{m}(t, \bar{x})=\tilde{\tilde{\Lambda}}^{-1} \Psi_{m-1}\left(t+1, \tilde{\Lambda} \bar{x}+\tilde{f}\left(t, \bar{x}, \Psi_{m-1}(t, \bar{x})\right)\right)- \\
-\tilde{\tilde{\Lambda}}^{-1} \tilde{\tilde{f}}\left(t, \bar{x}, \psi_{m-1}(t, \bar{x})\right), \quad m=1,2, \ldots .
\end{gathered}
$$

определим последовательность вектор-функции $\left\{\psi_{m}(t, \bar{x})\right\}$ и докажем, что в некоторой области $D *$ она равномерно сходится к вектор-функции $\psi(t, \bar{x})$, которая удовлетворяет указанным в теореме условиям и является решением системы уравнении (14).

Сначала покажем, что при достаточно малых $b_{*}<b$ и всех $m=0,1, \ldots$ выполняются оценки

$$
\begin{aligned}
& \left|\Psi_{m}(t, \bar{x})-\Psi_{m-1}(t, \bar{x})\right| \leq N_{0} \theta_{0}^{m-1}|\bar{x}|^{1+\alpha}, \\
& \left|\frac{\partial \Psi_{m}(t, \bar{x})}{\partial \bar{x}}-\frac{\partial \psi_{m-1}(t, \bar{x})}{\partial \bar{x}}\right| \leq N_{1} \theta_{1}^{m-1}|\bar{x}|^{\alpha}, \\
& \left|\frac{\partial \Psi_{m}\left(t, \bar{x}^{\prime}\right)}{\partial \bar{x}}-\frac{\partial \Psi_{m}\left(t, \bar{x}^{\prime \prime}\right)}{\partial \bar{x}}\right| \leq N\left|\bar{x}^{\prime}-\bar{x}^{\prime \prime}\right|^{\alpha},
\end{aligned}
$$

где $(t, \bar{x}),\left(t, \bar{x}^{\prime}\right),\left(t, \bar{x}^{\prime \prime}\right) \in D_{*}, N_{0}, N_{1}, N$ - некоторые положительные постоянные, $\quad \tilde{\tilde{\lambda}}_{*}^{-1} \tilde{\lambda}^{*+\alpha}<\theta_{0}<1, \quad \theta_{0}^{\alpha}<\dot{\theta}_{1}<1, \quad$ причем $\tilde{\lambda}^{*}=\max \left\{\left|\lambda_{i}\right|, i=1, \ldots, p\right\}$, $\tilde{\bar{\lambda}}_{*}=\min \left\{\left|\lambda_{i}\right|, i=p+1, \ldots, n\right\}$.

В самом деле, поскольку

$$
\Psi_{1}(t, \bar{x})=-\tilde{\tilde{\Lambda}}^{-1} \tilde{\tilde{f}}(t, \bar{x}, 0),
$$

то в силу условий 3,4 оценки $(16)-(18)$ выполняются при $\dot{m}=1$. П! гдположим, что оценки (16) - (18) доказаны уже для некоторого $m \geq 1$, и покажем, что они сохраняются при переходе от $m \mathbf{k} m+1$.

Действительно, в силу условий $1^{\prime}, 3,4$ и (16) - (18) при достаточно малых $|x| \leq b_{*}<b \quad\left(b_{*}<1\right)$ имеем

$$
\begin{aligned}
& \left|\tilde{f}\left(t, x^{\prime}, y^{\prime}\right)-\tilde{f}\left(t, x^{\prime \prime}, y^{\prime \prime}\right)\right| \leq L b_{*}^{\alpha}\left(\left|x^{\prime}-x^{\prime \prime}\right|+\left|y^{\prime}-y^{\prime \prime}\right|\right), \\
& \left|\tilde{\tilde{f}}\left(t, x^{\prime}, y^{\prime}\right)-\tilde{\tilde{f}}\left(t, x^{\prime \prime}, y^{\prime \prime}\right)\right| \leq L b_{*}^{\alpha}\left(\left|x^{\prime}-x^{\prime \prime}\right|+\left|y^{\prime}-y^{\prime \prime}\right|\right),
\end{aligned}
$$

где $\left(t, x^{\prime}, y^{\prime}\right),\left(t, x^{\prime \prime}, y^{\prime \prime}\right) \in D_{*}$

$$
\left|\psi_{m}(t, \bar{x})\right| \leq \frac{N_{0}}{1-\theta_{0}}|x|^{i+\alpha} \leq b,
$$




$$
\begin{gathered}
\left|\frac{\partial \psi_{m}(t, \bar{x})}{\partial \bar{x}}\right| \leq N|\bar{x}|^{\alpha}<N, \\
\left|\Psi_{m}\left(t, \bar{x}^{\prime}\right)-\psi_{m}\left(t, \bar{x}^{\prime \prime}\right)\right| \leq N b_{*}^{\alpha}\left|\bar{x}^{\prime}-\bar{x}^{\prime \prime}\right|, \\
|\bar{x}|,\left|\bar{x}^{\prime}\right|,\left|\bar{x}^{\prime \prime}\right| \leq b_{*} \\
\left|\tilde{\Lambda} \bar{x}+\tilde{f}\left(t, \bar{x}, \psi_{m}(t, \bar{x})\right)\right| \leq\left(\bar{\lambda}^{*}+\delta\right)|\bar{x}| \leq b_{*}, \\
\delta=\delta\left(b_{*}\right) \rightarrow 0 \text { при } b_{*} \rightarrow 0 .
\end{gathered}
$$

Тогда непосредственно из (15), (19) вытекает

$$
\begin{aligned}
& \left|\Psi_{m+1}(t, \bar{x})-\Psi_{m}(t, \bar{x})\right| \leq\left|\tilde{\tilde{\Lambda}}^{-1}\right| \mid \Psi_{m}\left(t+1, \tilde{\Lambda} \bar{x}+\tilde{f}\left(t, \bar{x}, \Psi_{m}(t, \bar{x})\right)\right)- \\
& -\Psi_{m}\left(t+1, \tilde{\Lambda} \bar{x}+\tilde{f}\left(t, \bar{x}, \Psi_{m-1}(t, \bar{x})\right)\right)|+| \tilde{\tilde{\Lambda}}^{-1}|| \Psi_{m}\left(t+1, \tilde{\Lambda} \bar{x}+\tilde{f}\left(t, \bar{x}, \Psi_{m-1}(t, \bar{x})\right)\right)- \\
& -\Psi_{m-1}\left(t+1, \tilde{\Lambda} \bar{x}+\tilde{f}\left(t, \bar{x}, \Psi_{m-1}(t, \bar{x})\right)\right)|+| \tilde{\tilde{\Lambda}}-1 \| \tilde{\tilde{f}}\left(t, \bar{x}, \Psi_{m}(t, \bar{x})\right)- \\
& -\tilde{\tilde{f}}\left(t, \bar{x}, \Psi_{m-1}(t, \bar{x})\right)\left|\leq \tilde{\bar{\lambda}}_{*}^{-1} N\right| \tilde{f}\left(t, \bar{x}, \Psi_{m}(t, \bar{x})\right)-\tilde{f}\left(t, \bar{x}, \Psi_{m-1}(t, \bar{x})\right) \mid+ \\
& +\overline{\bar{\lambda}}_{*}^{-1} N_{0} \theta_{0}^{m-1}\left|\tilde{\Lambda} \bar{x}+\tilde{f}\left(t, \bar{x}, \Psi_{m-1}(t, \bar{x})\right)\right|^{1+\alpha}+\tilde{\bar{\lambda}}_{*}^{-1} \mid \tilde{\tilde{f}}\left(t, \bar{x}, \Psi_{m}(t, \bar{x})\right)- \\
& -\left.\overline{\tilde{f}}\left(t, \bar{x}, \Psi_{m-1}(t, \bar{x})\right)\left|\leq \tilde{\bar{\lambda}}_{*}^{-1} N L b_{*}^{\alpha} N_{0} \theta_{0}^{m-1}\right| \bar{x}\right|^{1+\alpha}+ \\
& +\tilde{\tilde{\lambda}}_{*}^{-1} N_{0} \theta_{0}^{m-1}\left(\lambda^{*}+\delta\right)^{1+\alpha}|\bar{x}|^{1+\alpha}+\tilde{\bar{\lambda}}_{*}^{-1} L b_{*}^{\alpha} N_{0} \theta_{0}^{m-1}|\bar{x}|^{1+\alpha} \leq \\
& \leq N_{0} \theta_{0}^{m-1}\left[\tilde{\bar{\lambda}}_{*}^{-1} N L b_{*}^{\alpha}+\overline{\bar{\lambda}}_{*}^{-1}\left(\tilde{\bar{\lambda}}^{*}+\delta\right)^{1+\alpha}+\tilde{\bar{\lambda}}_{*}^{-1} L b_{*}^{\alpha}\right]|\bar{x}|^{1+\alpha} \text {. }
\end{aligned}
$$

Поскольку $\tilde{\tilde{\lambda}}_{*}^{-1} \tilde{\lambda}^{1+\alpha}<1$, то $\theta_{0}=\tilde{\bar{\lambda}}_{*}^{-1}\left(\tilde{\lambda}^{*}+\delta\right)^{1+\alpha}+\tilde{\tilde{\lambda}}_{*}^{-1} L N b_{*}^{\alpha}+\tilde{\bar{\lambda}}_{*}^{-1} L b_{*}^{\alpha}<1$ при достаточно малом $b_{*}^{\alpha}$ и, следовательно, оценка (16) сохраняется при переходе Oт $m \mathrm{k} m+1$.

Принимая во внимание (19) и соотношение

$$
\begin{aligned}
& \frac{\partial \Psi_{m+1}(t, \bar{x})}{\partial \bar{x}}=\tilde{\tilde{\Lambda}}^{-1} \frac{\partial \Psi_{m}}{\partial \bar{x}}\left(t+1, \tilde{\Lambda} \bar{x}+\tilde{f}\left(t, \bar{x}, \Psi_{m}(t, \bar{x})\right)\right)(\tilde{\Lambda}+ \\
& \left.+\frac{\partial \tilde{f}}{\partial \bar{x}}\left(t, \bar{x}, \Psi_{m}(t, \bar{x})\right)+\frac{\partial \tilde{f}}{\partial \psi}\left(t, \bar{x}, \Psi_{m}(t, \bar{x})\right) \frac{\partial \psi_{m}(t, \bar{x})}{\partial \bar{x}}\right)- \\
& -\tilde{\tilde{\Lambda}}^{-1} \frac{\partial \tilde{\tilde{f}}}{\partial \bar{x}}\left(t, \bar{x}, \Psi_{m}(t, \bar{x})\right)-\tilde{\tilde{\Lambda}}^{-1} \frac{\partial \overline{\tilde{f}}}{\partial \psi}\left(t, \bar{x}, \Psi_{m}(t, \bar{x})\right) \frac{\partial \Psi_{m}(t, x)}{\partial \bar{x}}
\end{aligned}
$$

HaxоднM

$$
\begin{aligned}
& \left|\frac{\partial \Psi_{m+1}(t, \bar{x})}{\partial \bar{x}}-\frac{\partial \Psi_{m}(t, \bar{x})}{\partial \bar{x}}\right| \leq\left|\overline{\bar{\Lambda}}^{-1}\right|\left[\left(\mid \frac{\partial \psi_{m}}{\partial \bar{x}}\left(t+1, \tilde{\Lambda} \bar{x}+\tilde{f}\left(t, \bar{x}, \Psi_{m}(t, \bar{x})\right)\right)-\right.\right. \\
& -\frac{\partial \psi_{m}}{\partial \bar{x}}\left(t+1, \tilde{\Lambda} \bar{x}+\tilde{f}\left(t, \bar{x}, \Psi_{m-1}(t, \bar{x})\right)\right)|+| \frac{\partial \Psi_{m}}{\partial \bar{x}}\left(t+1, \tilde{\Lambda} \bar{x}+\tilde{f}\left(t, \bar{x}, \Psi_{m-1}(t, \bar{x})\right)\right)-
\end{aligned}
$$




$$
\begin{aligned}
& \left.-\frac{\partial \Psi_{m-1}}{\partial \bar{x}}\left(t+1, \tilde{\Lambda} \bar{x}+\tilde{f}\left(t, \bar{x}, \Psi_{m-1}(t, \bar{x})\right)\right) \mid\right) \mid \tilde{\Lambda}+\frac{\partial}{\partial \bar{x}} \tilde{f}\left(t, \bar{x}, \Psi_{m}(t, \bar{x})\right)+ \\
& +\frac{\partial \tilde{f}}{\partial \psi}\left(t, \bar{x}, \Psi_{m}(t, \bar{x})\right) \frac{\partial \psi_{m}(t, \bar{x})}{\partial \bar{x}}|+| \frac{\partial \psi_{m-1}}{\partial \bar{x}}\left(t+1, \tilde{\Lambda} \bar{x}+\tilde{f}\left(t, \bar{x}, \Psi_{m-1}(t, \bar{x})\right)\right) \mid \times \\
& \times\left(\left|\frac{\partial \tilde{f}}{\partial \bar{x}}\left(t, \bar{x}, \Psi_{m}(t, \bar{x})\right)-\frac{\partial \tilde{f}}{\partial \bar{x}}\left(t, \bar{x}, \Psi_{m-1}(t, \bar{x})\right)\right|+\mid \frac{\partial \tilde{f}}{\partial \psi}\left(t, \bar{x}, \Psi_{m}(t, \bar{x})\right) \| \frac{\partial \Psi_{m}(t, \bar{x})}{\partial \bar{x}}-\right. \\
& \left.\left.-\frac{\partial \psi_{m-1}(t, \bar{x})}{\partial \bar{x}}|+| \frac{\partial \psi_{m-1}(t, \bar{x})}{\partial \bar{x}}\left|\frac{\partial \tilde{f}}{\partial \psi}\left(t, \bar{x}, \psi_{m}(t, \bar{x})\right)-\frac{\partial \tilde{f}}{\partial \psi}\left(t, \bar{x}, \Psi_{m-1}(t, \bar{x})\right)\right|\right)\right]+ \\
& +|\tilde{\tilde{\Lambda}}-1|\left|\frac{\partial \tilde{\tilde{f}}}{\partial \bar{x}}\left(t, \bar{x}, \Psi_{m}(t, \bar{x})\right)-\frac{\partial \tilde{\tilde{f}}}{\partial \bar{x}}\left(t, \bar{x}, \Psi_{m-1}(t, \bar{x})\right)\right|+\left|\tilde{\tilde{\Lambda}}^{-1}\right|\left(\left|\frac{\partial \tilde{\tilde{f}}}{\partial \psi}\left(t, \bar{x}, \Psi_{m}(t, \bar{x})\right)\right| \times\right. \\
& \times\left|\frac{\partial \Psi_{m}(t, \bar{x})}{\partial \bar{x}}-\frac{\partial \Psi_{m-1}(t, \bar{x})}{\partial \bar{x}}\right|+\left|\frac{\partial \psi_{m-1}(t, \bar{x})}{\partial \bar{x}}\right| \mid \frac{\partial \tilde{\tilde{f}}}{\partial \psi}\left(t, \bar{x}, \Psi_{m}(t, \bar{x})\right)- \\
& \left.-\frac{\partial \tilde{\tilde{f}}}{\partial \psi}\left(t, \bar{x}, \Psi_{m-1}(t, \bar{x})\right) \mid\right) \leq \tilde{\tilde{\lambda}}_{*}^{-1}\left[\left(N\left|\tilde{f}\left(t, \bar{x}, \Psi_{m}(t, \bar{x})\right)-\tilde{f}\left(t, \bar{x}, \psi_{m-1}(t, \bar{x})\right)\right|+\right.\right. \\
& \left.+N_{1} \theta_{1}^{m-1}\left|\bar{\Lambda} \bar{x}+\bar{f}\left(t, \bar{x}, \psi_{m-1}^{\prime}(t, \bar{x})\right)\right|^{\alpha}\right)\left(\tilde{\lambda}^{*}+L\left(|\bar{x}|+\left|\psi_{m}(t, \bar{x})\right|\right)^{\alpha}+\right. \\
& \left.+L\left(|\bar{x}|+\left|\Psi_{m}(t, \bar{x})\right|\right)^{\alpha} N|\bar{x}|^{\alpha}\right)+N\left|\tilde{\Lambda} \bar{x}+\dot{\tilde{f}}\left(t, \bar{x}, \Psi_{m-1}(t, \bar{x})\right)\right|^{\alpha} \times \\
& \times\left(L\left|\Psi_{m}(t, \bar{x})-\Psi_{m-1}(t, \bar{x})\right|^{\alpha}+L\left(|\bar{x}|+\left|\Psi_{m}(t, \bar{x})\right|\right)^{\alpha} N_{1} \theta_{1}^{m-1}|\bar{x}|^{\alpha}+\right. \\
& \left.\left.+N|\bar{x}|^{\alpha} L\left|\Psi_{m}(t, \bar{x})-\Psi_{m-1}(t, \bar{x})\right|^{\alpha}\right)\right]+\tilde{\bar{\lambda}}_{*}^{-1} L\left|\Psi_{m}(t, \bar{x})-\Psi_{m-1}(t, \bar{x})\right|^{\alpha}+ \\
& +\tilde{\bar{\lambda}}_{*}^{-1}\left(L\left(|\bar{x}|+\left|\Psi_{m}(t, \bar{x})\right|\right)^{\alpha} N_{1} \theta_{1}^{m-1}|\bar{x}|^{\alpha}+N|\bar{x}|^{\alpha} L \mid \Psi_{m}(t, \bar{x})-\right. \\
& \left.-\left.\Psi_{m-1}(t, \bar{x})\right|^{\alpha}\right) \leq \tilde{\tilde{\lambda}}_{*}^{-1}\left[\left(N L b_{*}^{\alpha} N_{0} \theta_{0}^{m-1}|\bar{x}|^{1+\alpha}+N_{1} \theta_{1}^{m-1}\left(\tilde{\lambda}^{*}+\delta\right)^{\alpha}|\bar{x}|^{\alpha}\right) \times\right. \\
& \times\left(\tilde{\lambda}^{*}+L\left(|\bar{x}|+\frac{N_{0}}{1-\theta_{0}}|\bar{x}|^{1+\alpha}\right)^{\alpha}+L\left(|\bar{x}|+\frac{N_{0}}{1-\theta_{0}}|\bar{x}|^{1+\alpha}\right)^{\alpha} N|\bar{x}|^{\alpha}\right)+ \\
& +N\left(\bar{\lambda}^{*}+\delta\right)^{\alpha}|\bar{x}|^{\alpha}\left(L N_{0}^{\alpha} \theta_{0}^{\alpha(m-1)}|\bar{x}|^{\alpha(1+\alpha)}+L\left(|\bar{x}|+\frac{N_{0}}{1-\theta_{0}}|\bar{x}|^{1+\alpha}\right)^{\alpha} \times\right. \\
& \left.\left.\times N_{1} \theta_{1}^{m-1}|\bar{x}|^{\alpha}+N L|\bar{x}|^{\alpha} N_{0}^{\alpha} \theta_{0}^{\alpha(m-1)}|\bar{x}|^{\alpha(1+\alpha)}\right)\right]+\tilde{\bar{\lambda}}_{*}^{-1} L N_{0}^{\alpha} \theta_{0}^{\alpha(m-1)}|\bar{x}|^{\alpha(1+\alpha)}+ \\
& +\tilde{\bar{\lambda}}_{*}^{-1}\left(L N_{1} \theta_{1}^{m-1}|\bar{x}|^{\alpha}\left(|\bar{x}|+\frac{N_{0}}{1-\theta_{0}}|\bar{x}|^{1+\alpha}\right)^{\alpha}+L N N_{0}^{\alpha} \dot{\theta}_{0}^{\alpha(m-1)}|\bar{x}|^{\alpha}|\bar{x}|^{\alpha(1+\alpha)}\right) \leq
\end{aligned}
$$




$$
\begin{gathered}
\leq N_{1} \theta_{1}^{m-1} \overline{\bar{\lambda}}_{*}^{-1}\left[( ( \overline { \lambda } ^ { * } + \delta ) ^ { \alpha } + L N _ { 0 } N N _ { 1 } ^ { - 1 } b _ { * } ^ { 1 + \alpha } ( \frac { \theta _ { 0 } } { \theta _ { 1 } } ) ^ { m - 1 } ) \left(\tilde{\lambda}^{*}+\right.\right. \\
\left.+L\left(b_{*}+\frac{N_{0}}{1-\theta_{0}} b_{*}^{1+\alpha}\right)^{\alpha}\left(1+N b_{*}^{\alpha}\right)\right)+N\left(\bar{\lambda}^{*}+\delta\right)^{\alpha}\left(L N_{0}^{\alpha} N_{1}^{-1}\left(\frac{\theta_{0}^{\alpha}}{\theta_{1}}\right)^{m-1} \times\right. \\
\left.\times b_{*}^{\alpha(1+\alpha)}+L\left(b_{*}+\frac{N_{0}}{1-\theta_{0}} b_{*}^{1+\alpha}\right)^{\alpha} b_{*}^{\alpha}+L N_{0}^{\alpha} N N_{1}^{-1}\left(\frac{\theta_{0}^{\alpha}}{\theta_{1}}\right)^{m-1} b_{*}^{\alpha(2+\alpha)}\right)+ \\
+L N_{0}^{\alpha} N_{1}^{-1}\left(\frac{\theta_{0}^{\alpha}}{\theta_{1}}\right)^{m-1} b_{*}^{\alpha^{2}}+L\left(b_{*}+\frac{N_{0}}{1-\theta_{0}} b_{*}^{1+\alpha}\right)^{\alpha}+ \\
\left.+L N_{0}^{\alpha} N N_{1}^{-1}\left(\frac{\theta_{0}^{\alpha}}{\theta_{1}}\right)^{m-1} b_{*}^{\alpha(1+\alpha)}\right]|\bar{x}|^{\alpha} .
\end{gathered}
$$

Таким образом, поскольку $\tilde{\bar{\lambda}}_{*}^{-1} \tilde{\lambda}^{*^{1+\alpha}}<\theta_{0} \leq \theta_{0}^{\alpha}<\theta_{1}<1$, то из последнего соотношения при достаточно малом $b$ * получаем

$$
\left|\frac{\partial \Psi_{m+1}(t, \bar{x})}{\partial \bar{x}}-\frac{\partial \psi_{m}(t, \bar{x})}{\partial \bar{x}}\right| \leq N_{1} \theta_{I}^{m}|\bar{x}|^{\alpha}
$$

т. е. оценка (17) справедлива при $m+1$.

Аналогично находим

$$
\begin{aligned}
& \left|\frac{\partial \Psi_{m+1}\left(t, \bar{x}^{\prime}\right)}{\partial \bar{x}}-\frac{\partial \Psi_{m+1}\left(t, \bar{x}^{\prime \prime}\right)}{\partial \bar{x}}\right| \leq \tilde{\tilde{\lambda}}_{*}^{-1} \mid \frac{\partial \Psi_{m}}{\partial \bar{x}}\left(t+1, \bar{\Lambda}^{\prime}+\tilde{f}\left(t, \bar{x}^{\prime}, \Psi_{m}\left(t, \bar{x}^{\prime}\right)\right)\right)- \\
& -\frac{\partial \psi_{m}}{\partial \bar{x}}\left(t+1, \tilde{\Lambda} \bar{x}^{\prime \prime}+\tilde{f}\left(t, \bar{x}^{\prime \prime}, \Psi_{m}\left(t, \bar{x}^{\prime \prime}\right)\right)\right) \| \tilde{\Lambda}+\frac{\partial \tilde{f}}{\partial \bar{x}}\left(t, \bar{x}^{\prime}, \psi_{m}\left(t, \bar{x}^{\prime}\right)\right)+ \\
& +\frac{\partial \tilde{f}}{\partial \psi}\left(t, \bar{x}^{\prime}, \Psi_{m}\left(t, \bar{x}^{\prime}\right)\right) \frac{\partial \Psi_{m}\left(t, \bar{x}^{\prime}\right)}{\partial \bar{x}^{\prime}} \mid+ \\
& +\tilde{\bar{\lambda}}_{*}^{-1}\left|\frac{\partial \Psi_{m}}{\partial \bar{x}}\left(t+1, \tilde{\Lambda} \bar{x}^{\prime \prime}+\tilde{f}\left(t, \bar{x}^{\prime \prime}, \psi_{m}\left(t, \bar{x}^{\prime \prime}\right)\right)\right)\right|\left(\mid \frac{\partial \bar{f}}{\partial \bar{x}}\left(t, \bar{x}^{\prime}, \Psi_{m}\left(t, \bar{x}^{\prime}\right)\right)-\right. \\
& -\frac{\partial \tilde{f}}{\partial \bar{x}}\left(t, \bar{x}^{\prime \prime}, \Psi_{m}\left(t, \bar{x}^{\prime \prime}\right)\right)|+| \frac{\partial \Psi_{m}\left(t, \bar{x}^{\prime}\right)}{\partial \bar{x}} \| \frac{\partial \tilde{f}}{\partial \psi}\left(t, \bar{x}^{\prime}, \Psi_{m}\left(t, \bar{x}^{\prime}\right)\right)- \\
& -\frac{\partial \tilde{f}}{\partial \psi}\left(t, \bar{x}^{\prime \prime}, \Psi_{m}\left(t, \bar{x}^{\prime \prime}\right)\right)|+| \frac{\partial \tilde{f}}{\partial \psi}\left(t, \bar{x}^{\prime \prime}, \psi_{m}\left(t, \bar{x}^{\prime \prime}\right)\right) \mid \frac{\partial \psi_{m}\left(t, \bar{x}^{\prime}\right)}{\partial \bar{x}}- \\
& \left.-\frac{\partial \Psi_{m}\left(t, \bar{x}^{\prime \prime}\right)}{\partial \bar{x}} \mid\right)+\tilde{\tilde{\lambda}}_{*}^{-1}\left|\frac{\partial \tilde{\tilde{f}}}{\partial \bar{x}}\left(t, \bar{x}^{\prime}, \Psi_{m}\left(t, \bar{x}^{\prime}\right)\right)-\frac{\partial \tilde{\tilde{f}}}{\partial \bar{x}}\left(t, \bar{x}^{\prime \prime}, \Psi_{m}\left(t, \bar{x}^{\prime \prime}\right)\right)\right|+ \\
& +\tilde{\bar{\lambda}}_{*}^{-1}\left(\left|\frac{\partial \Psi_{m}\left(t, \bar{x}^{\prime}\right)}{\partial \bar{x}}\right|\left|\frac{\partial \tilde{\tilde{f}}}{\partial \psi}\left(t, \bar{x}^{\prime}, \Psi_{m}\left(t, \bar{x}^{\prime}\right)\right)-\frac{\partial \overline{\tilde{f}}}{\partial \psi}\left(t, \bar{x}^{\prime \prime}, \Psi_{m}\left(t, \bar{x}^{\prime \prime}\right)\right)\right|+\right.
\end{aligned}
$$




$$
\begin{aligned}
& \left.\quad+\left|\frac{\partial \tilde{\tilde{f}}\left(t, \bar{x}^{\prime \prime}, \Psi_{m}\left(t, \bar{x}^{\prime \prime}\right)\right)}{\partial \psi}\right|\left|\frac{\partial \psi_{m}\left(t, \bar{x}^{\prime}\right)}{\partial \bar{x}}-\frac{\partial \psi_{m}\left(t, \bar{x}^{\prime \prime}\right)}{\partial \bar{x}}\right|\right) \leq \\
& \leq \tilde{\bar{\lambda}}_{*}^{-1} N\left(\tilde{\lambda}^{*}\left|\bar{x}^{\prime}-\bar{x}^{\prime \prime}\right|+L b_{*}^{\alpha}\left(\left|\bar{x}^{\prime}-\bar{x}^{\prime \prime}\right|+N b_{*}^{\alpha}\left|\bar{x}^{\prime}-\bar{x}^{\prime \prime}\right|\right)\right)^{\alpha}\left(\bar{\lambda}^{*}+\right. \\
& \left.+L\left(\left|\bar{x}^{\prime}\right|+N b_{*}^{\alpha}\left|\bar{x}^{\prime}\right|\right)^{\alpha}+L\left(\left|\bar{x}^{\prime}\right|+N b_{*}^{\alpha}\left|\bar{x}^{\prime}\right|\right)^{\alpha} N\left|\bar{x}^{\prime}\right|^{\alpha}\right)+ \\
& +\tilde{\tilde{\lambda}}_{*}^{-1} N\left(\bar{\lambda}^{*}\left|\bar{x}^{\prime \prime}\right|+L b_{*}^{\alpha}\left(\left|\bar{x}^{\prime \prime}\right|+N b_{*}^{\alpha}\left|\bar{x}^{\prime \prime}\right|\right)\right)^{\alpha}\left(L \left(\left|\bar{x}^{\prime}-\bar{x}^{\prime \prime}\right|+\right.\right. \\
& \left.+N b_{*}^{\alpha}\left|\bar{x}^{\prime}-\bar{x}^{\prime \prime}\right|\right)^{\alpha}+N\left|\bar{x}^{\prime}\right|^{\alpha} L\left(\left|\bar{x}^{\prime}-\bar{x}^{\prime \prime}\right|+N b_{*}^{\alpha}\left|\bar{x}^{\prime}-\bar{x}^{\prime \prime}\right|\right)^{\alpha}+ \\
& \left.+L\left(\left|\bar{x}^{\prime \prime}\right|+N b_{*}^{\alpha}\left|\bar{x}^{\prime \prime}\right|\right)^{\alpha} N\left|\bar{x}^{\prime}-\bar{x}^{\prime \prime}\right|^{\alpha}\right)+\tilde{\tilde{\lambda}}_{*}^{-1} L\left(\left|\bar{x}^{\prime}-\bar{x}^{\prime \prime}\right|+\right. \\
& \left.+N b_{*}^{\alpha}\left|\bar{x}^{\prime}-\bar{x}^{\prime \prime}\right|\right)^{\alpha}+\overline{\tilde{\lambda}}_{*}^{-1}\left(N\left|\bar{x}^{\prime}\right|^{\alpha} L\left(\left|\bar{x}^{\prime}-\bar{x}^{\prime \prime}\right|+N b_{*}^{\alpha}\left|\bar{x}^{\prime} \cdot-\bar{x}^{\prime \prime}\right|\right)^{\alpha}+\right. \\
& \left.+L\left(\left|\bar{x}^{\prime \prime}\right|+N b_{*}^{\alpha}\left|\bar{x}^{\prime \prime}\right|\right)^{\alpha} N\left|\bar{x}^{\prime}-\bar{x}^{\prime \prime}\right|^{\alpha}\right) \leq N\left|\bar{x}^{\prime}-\bar{x}^{\prime \prime}\right|^{\alpha} \tilde{\tilde{\lambda}_{*}^{-1}}\left[\left(\tilde{\lambda}^{*}+\right.\right. \\
& \left.+L b_{*}^{\alpha}\left(1+N b_{*}^{\alpha}\right)\right)^{\alpha}\left(\tilde{\lambda}^{*}+L\left(b_{*}+N b_{*}^{1+\alpha}\right)^{\alpha}\left(1+N b_{*}^{\alpha}\right)\right)+ \\
& +\left(\tilde{\lambda}^{*} b_{*}+L b_{*}^{\alpha}\left(b_{*}+N b_{*}^{1+\alpha}\right)\right)^{\alpha}\left(L\left(1+N b_{*}^{\alpha}\right)^{\alpha}+L N b_{*}^{\alpha}\left(1+N b_{*}^{\alpha}\right)^{\alpha}+\right. \\
& \left.+L N\left(b_{*}+N b_{*}^{1+\alpha}\right)^{\alpha}\right)+L N^{-1}\left(1+N b_{*}^{\alpha}\right)^{\alpha}+L b_{*}^{\alpha}\left(1+N b_{*}^{\alpha}\right)^{\alpha}+
\end{aligned}
$$

Отсюда следует, что при достаточно малом $b_{*}$ и достаточно большом $N$ имеем

$$
,\left|\frac{\partial \psi_{m+1}\left(t, \bar{x}^{\prime}\right)}{\partial \bar{x}}-\frac{\partial \psi_{m+1}\left(t, \bar{x}^{\prime \prime}\right)}{\partial \bar{x}}\right| \leq N\left|\bar{x}^{\prime}-\bar{x}^{\prime \prime}\right|^{\alpha},
$$

т. е. оценка (18) выполняется при замене $m \cdot$ на $m+1$. Таким образом, при достаточно малом $b$ * и достаточно большом $N$ оценки $(16)-(18)$ справедливы при всех $m \geq 0$.

Непосредственно из (16) - (18) вытекает, что последовательность векторфункций $\left\{\psi_{m}(t, \bar{x})\right\}$, определенных соотношениями (15), равномерно схуодится при $|\bar{x}| \leq b$ * к вектор-функции $\psi(t, \bar{x})$, которая удовлетворяет условиям, указанным в теореме 2 . Переходя в (15) к пределу при $m \rightarrow \infty$, легко убедиться, что вектор-функция $\psi(t, \bar{x})=\lim _{m \rightarrow \infty} \psi_{m}(t, \bar{x})$ является решением системы уравнений (14). Теорема доказана.

В силу теоремы 2 исследование окрестности тривиального решения системы уравнений (10) сводится к исследованию окрестности тривиального решения системы (13). Покажем сначала, что если $(\bar{x}(t), \bar{y}(t))$ - некоторое непрерывное при $t \geq 0$ решение системы уравнений (13), находящееся в окрестности ее трнвиального решения $(0,0)$, то $\bar{y}(t) \equiv 0$. Дећствительно, пусть это не так, т. е. имеется некоторое непрерывное при $t \geq 0$ решение $(\bar{x}(t), \bar{y}(t))$ системы (13), находящееся в некоторой окрестности ее тривиального решения и такое, что $\bar{y}(t) \neq 0$. Тогда в силу условий $1^{\prime}, 3,4$ и $\overline{\bar{f}}(t, \bar{x}, 0) \equiv 0$ имеем 


$$
|\overline{\bar{f}}(t, \bar{x}, \bar{y})| \leq \varepsilon|\bar{y}| .
$$

где $\varepsilon \rightarrow 0$ при $|\bar{x}|,|\bar{y}| \rightarrow 0$, и из (13) получаем

$$
|\bar{y}(t+1)| \geq\left(\tilde{\tilde{\lambda}}_{*}-\varepsilon\right)|\bar{y}(t)|
$$

Поскольку $\tilde{\bar{\lambda}}_{*}>1$, то $\tilde{\bar{\lambda}}_{*}-\varepsilon>1$ при достаточно малом $\varepsilon$ и из последнего соотношения вытекает

$$
|\bar{y}(t+m)| \geq\left(\tilde{\tilde{\lambda}}_{*}-\varepsilon\right)^{m}|\bar{y}(t)|, \quad m \geq 1
$$

т. е. $|\bar{y}(t)| \rightarrow \infty$ при $t \rightarrow \infty$. Полученное противоречие доказывает, что $|\bar{y}(t)| \equiv$ $\equiv 0$. Следовательно, для построения всех непрерывных при $t \geq 0$ решений системы уравненни (13), находящихся в окрестности ее тривиального решения, достаточно построить все непрерывные при $t \geq 0$ решения системы уравнений

$$
\bar{x}(t+1)=\bar{\Lambda} \bar{x}(t)+\bar{f}(t, \bar{x}(t), 0)
$$

находящиеся в окрестности ее тривиального решения $\bar{x}(t)=0$. Так как для системы (20) выполняются все условия теоремы 1 , то ее общее непрерывное при $t \geq 0$ решение имеет вид (9), где $n=p$. Принимая во внимание (11), получаем, что если $(\bar{x}(t), \bar{y}(t))$ - некоторое непрерывное при $t \geq 0$ решение системы уравнении (10), находящееся в достаточно малой окрестности ее тривиального решения $(0,0)$, то $x(t) \doteq z(t)+\gamma^{-1}(t, z(t)), \quad y(t)=\psi\left(t, z(t)+\gamma^{-1}(t, z(t))\right)$, где $z(t)=\left(\left|\lambda_{1}\right|^{t} \omega_{1}(t), \ldots,\left|\lambda_{p}\right|^{t} \omega_{p}(t)\right)$ и $\omega_{i}(t), i=1, \ldots, p$, - некоторые непрерывные функции, удовлетворяющие условиям $\omega_{i}(t+1)=\operatorname{sign} \lambda_{i} \omega_{i}(t), i=1, \ldots, p$.

1. Birkhoff G.D., Trjitzinsky W.J. Analitic theory of singular difference equations // Acta math. 1932. - 60. - P. 1 - 89.

2. Harris Jr., W.A., Sibuya $Y$. General solution of nonlinear difference equations // Trans. Amer. Math. Soc. - 1965. - 115. P. 62 - 75.

3. Takano By. $K$. General solution of a nonlinear difference equations of Briot - Bouquet type $/ /$ Funkc. ekvacioj. - 1971. - 13, № 3. - P. $179-198$.

4. Takano By. $K$. Solution containing arbitrary periodic functions of systems of nonlinear difference equations // Ibid. - 1973. -13, N 2. - P. 137 - 164.

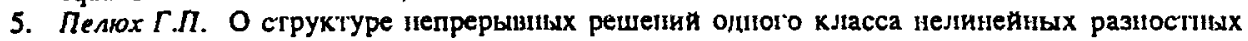
уравнепия // Дифферепщ. уравнеІия. - 1994. - 30. N 6. - С. 1083 - 1085.

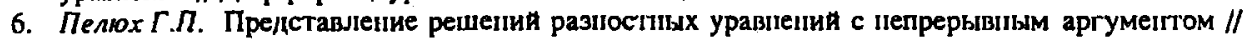
Там же. $-1996 .-32, N^{2} 2 .-$ C. $304-312$. 